\title{
Alleviation of Chronic Cancer Pain by Adrenal Medullary Transplants in the Spinal Subarachnoid Space
}

\author{
Jacqueline Sagen ${ }^{1}$, George D. Pappas ${ }^{1}$ and Alon P. Winnie ${ }^{2}$ \\ ${ }^{1}$ Department of Anatomy and Cell Biology and ${ }^{2}$ Department of Anesthesiology, University of Illinois at \\ Chicago, 808 S. Wood St., Chicago, IL 60612, USA
}

Work in our laboratory over the past several years has demonstrated that the transplantation of adrenal medullary chromaffin cells into the spinal subarachnoid space can markedly reduce pain in several rodent models without the development of tolerance. Adrenal medullary chromaffin cells were selected since they produce high levels of both opioid peptides and catecholamines, agents which independently, and possibly synergistically, reduce pain when injected locally into the spinal subarachnoid space. The present study was an initial attempt to assess the potential for this approach to alleviate chronic pain in man.

Approval for this preliminary study was obtained from the Institutional Review Board of the University of Illinois. Consenting patients selected for the study were suffering from pain associated with terminal cancer with reduced pain control by escalating doses of narcotic analgesics. Human adrenal glands were obtained from the Regional Organ Bank of Illinois, and adrenal medullary tissue was prepared for transplantation in our laboratory. Using sterile techniques with the patient in the lateral decubitus position, lumbar puncture using a 14-gauge Tuohy needle was performed. After aspiration of sufficient CSF for culture, cytology, and biochemical assays, approximately $1 \mathrm{ml}$ of suspended adrenal medullary tissue was injected and the needle withdrawn. Cyclosporine A (10 $\mathrm{mg} / \mathrm{kg}$ orally) was administered for two weeks beginning one day prior to the procedure.

Pain levels were determined using a Visual Analog pain scale prior to and at weekly intervals following the procedure. In addition, records of daily analgesics consumed were kept by the patient or patient's family. When possible, CSF samples were collected via lumbar puncture at several intervals following the implantation for cytological and biochemical analysis.

Patient 1 was a 61 year old female with a six year history of carcinoma of the colon. As her disease advanced, she developed increasing back and lower extremity pain which became intractable, despite escalating doses of narcotics. Following transplantation, progressive decreases in pain scores and narcotic intake were reported, and the patient remained pain free until her death $4 \frac{1}{2}$ months following the procedure. Her CSF met-enkephalin levels were elevated 6-fold 1 month following the procedure.

Patient 2 was a 69 year old white male with a four-year history of carcinoma of the colon. His pain became progressively worse and more resistant to analgesic medications. The pain was highly position dependent, in that it became unbearable within a short period of time when he attempted to sit up. An epidural catheter for narcotic delivery provided significant improvement in pain relief, but became infected and had to be removed. Progressive reduction in pain scores followed adrenal medullary transplantation, and the patient continued to be pain free until his death 11 months later. In addition, the patient's activity, indicated by time spent out of bed, improved significantly following transplantation.

Patient 3 was a 49 year old Hispanic female with a three-year history of carcinoma of the left breast treated by mastectomy followed by chemotherapy. Two years later she began to complain of pain in her low back, right hip, and buttocks which was becoming unresponsive to narcotic analgesics. During the first month following adrenal medullary implantation, the patient's pain scores decreased dramatically. However, at this point the patient began to 
complain of progressive weakness of her lower extremities and a CT scan/myelogram revealed a spinal block due to metastasis. Since that time the patient had a recurrence of her pain.

Patient 4 was a 57 year old Hispanic male with a three-year history of colon carcinoma, initially treated by surgical resection followed by chemotherapy and radiation therapy. He did well until he developed pain in the sacral area which became unresponsive to increasing doses of narcotic analgesics. Following implantation, the patient's pain scores fell rapidly, and by the end of three weeks, the patient had eliminated all narcotic intake. CSF levels of both norepinephrine and met-enkephalin were significantly increased. At the end of the 10th month following the procedure, the patient still remained pain free.
At least three of the patients in this preliminary clinical study obtained significant, longlasting pain relief following adrenal medullary transplantation in the spinal subarachnoid space. A fourth patient obtained similar relief early, but her pain returned, possibly due to complications resulting from spinal cord compression secondary to metastasis. The mechanism for pain reduction by adrenal medullary transplants may be via increases in opioid peptide and catecholamine levels. The encouraging results of this initial pilot study suggest that adrenal medullary transplantation may provide a unique and effective approach in the management of pain in man.

Supported by NIH Grants NS25054 and NS28931. 

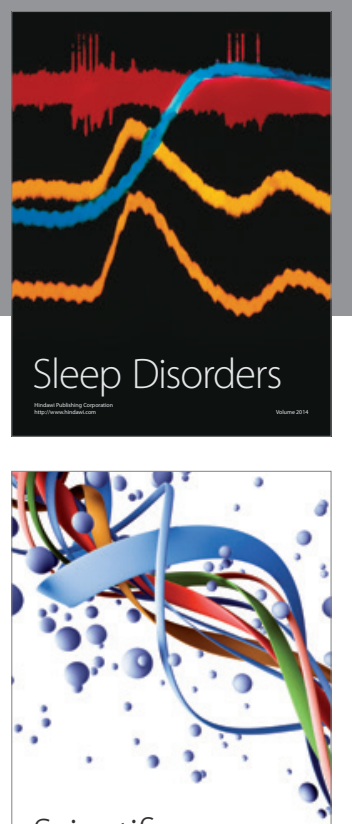

Scientifica
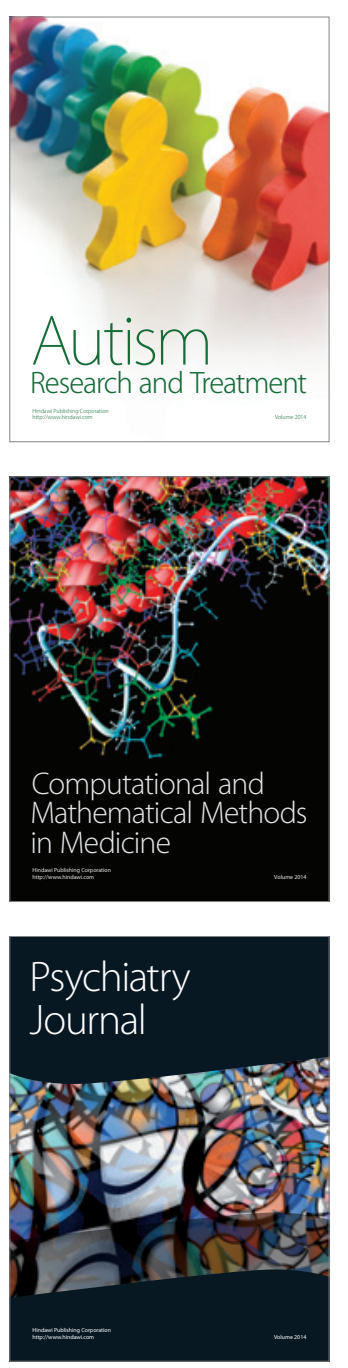
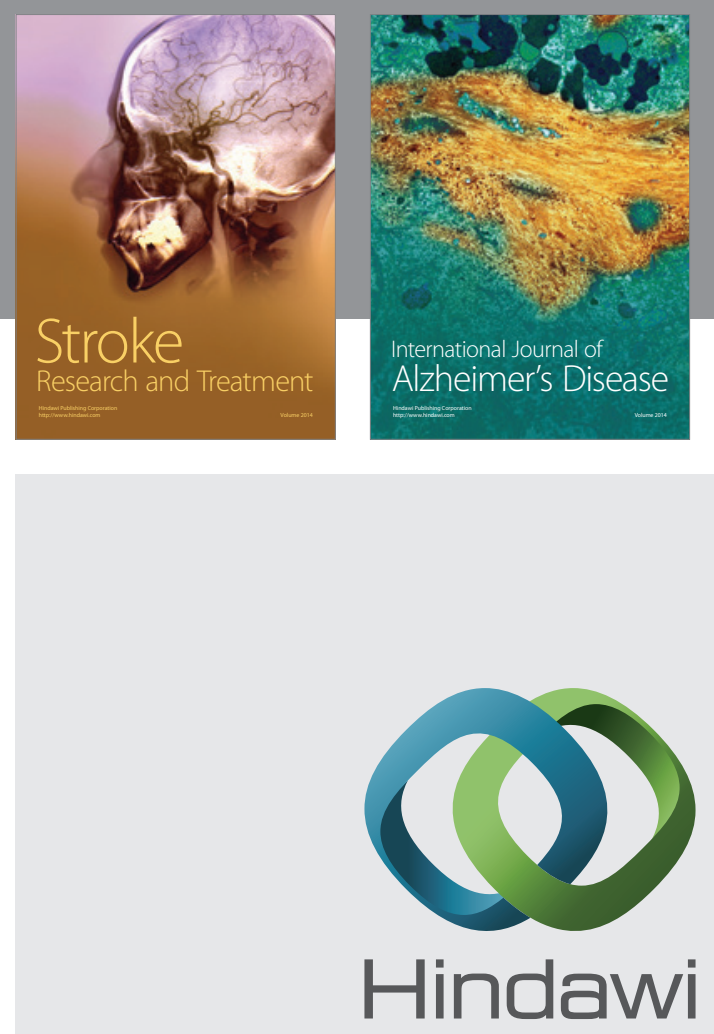

Submit your manuscripts at

http://www.hindawi.com
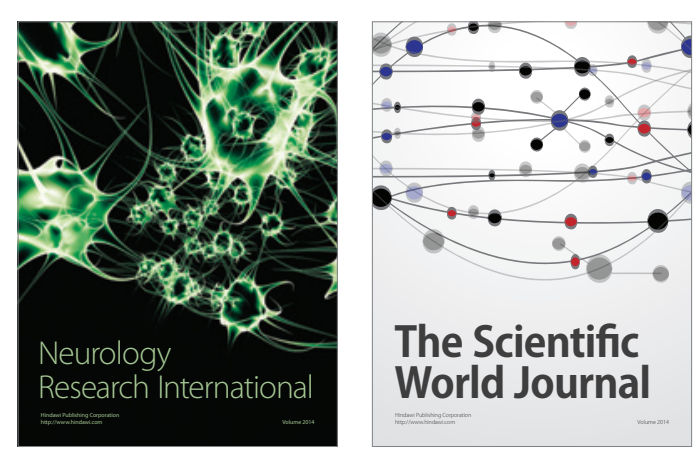

The Scientific World Journal

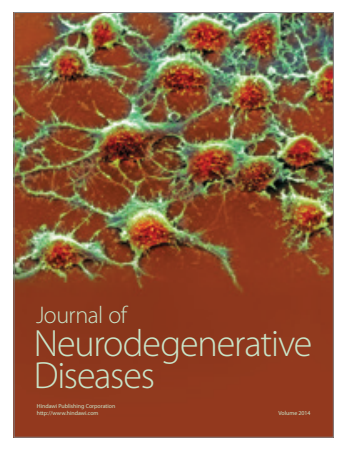

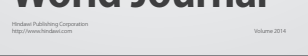

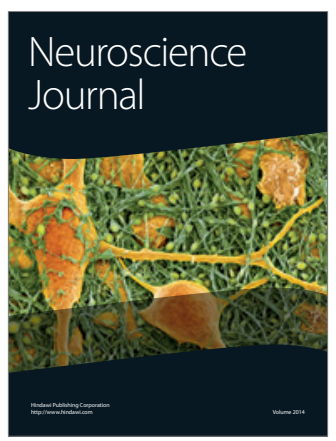

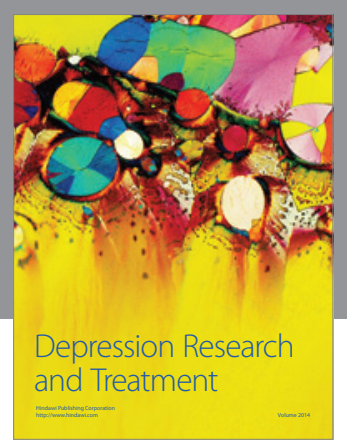
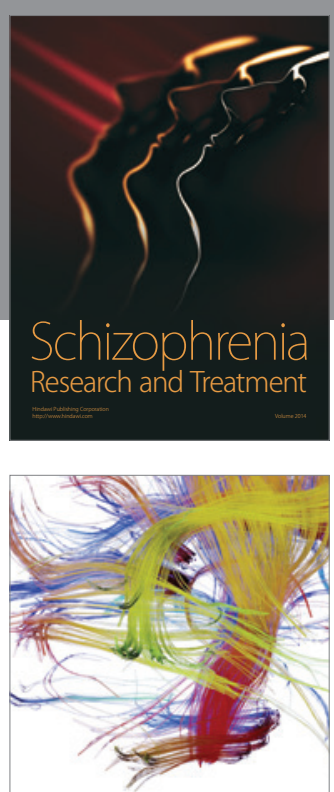

Brain Science

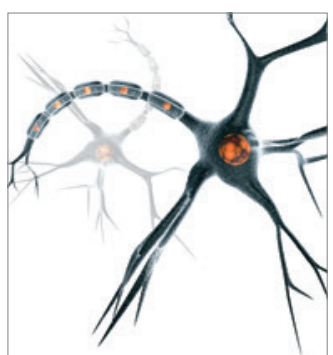

Neural Plasticity
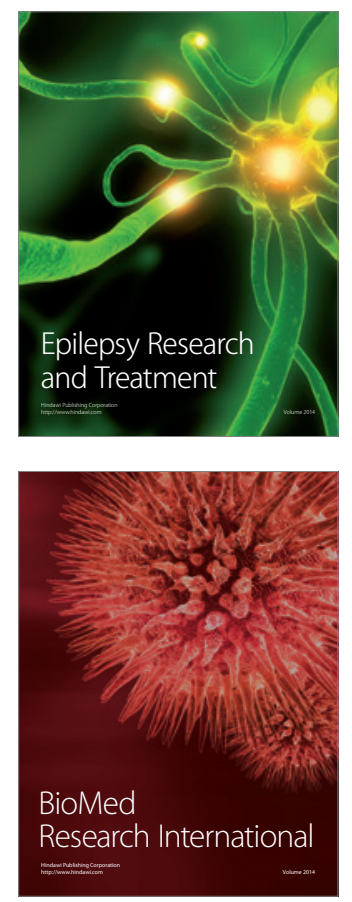

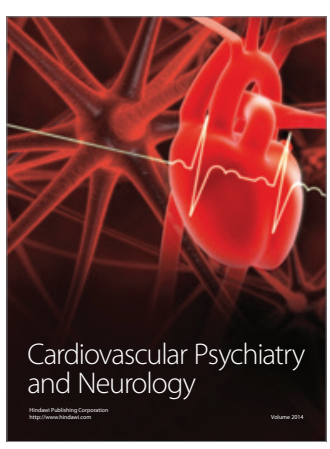

Parkinson's

Disease
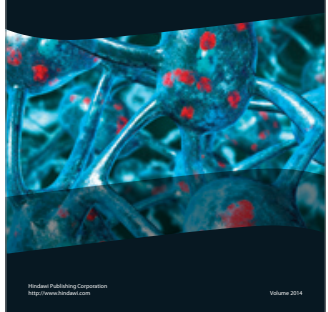